\title{
Coherent quantum switch driven by optimized laser pulses
}

\author{
E. Räsänen ${ }^{\mathrm{a}, \mathrm{b}, 1}$, A. Castro $^{\mathrm{a}, \mathrm{b}}$ J. Werschnik $^{\mathrm{c}, \mathrm{a}, \mathrm{b}}$ A. Rubio ${ }^{\mathrm{d}, \mathrm{a}, \mathrm{b}}$ and E. K. U. Gross ${ }^{\mathrm{a}, \mathrm{b}}$ \\ a Institut für Theoretische Physik, Freie Universität Berlin, Arnimallee 14, D-14195 Berlin, Germany \\ ${ }^{\mathrm{b}}$ European Theoretical Spectroscopy Facility (ETSF) \\ ' JENOPTIK Laser, Optik, Systeme GmbH, Göschwitzer Str. 25, D-07745 Jena, Germany \\ ${ }^{\mathrm{d}}$ Departamento de Física de Materiales, Facultad de Químicas Universidad del País Vasco, Centro Mixto CSIC-UPV, \\ Donostia International Physics Center (DIPC), E-20018 Donostia-San Sebastián, Spain
}

\begin{abstract}
We present two models of coherent quantum switches constructed from a single-electron quantum ring and a double quantum dot, respectively. The systems are driven by picosecond laser pulses obtained using quantum optimal control theory. The optimized pulses generate electron-current flips in the ring (magnetic switch) and electron transport in the double dot (charge switch) in significantly shorter times and higher accuracies than previously used finite-length continuous waves. This opens applicable paths into coherent single-qubit gates operated by terahertz lasers.
\end{abstract}

Key words: optimal control theory, quantum ring, double quantum dot, qubit PACS: 78.67.-n, 73.23.Ra, 78.20.Bh

Coherent quantum control of nanoscale systems has been under recent extensive studies both experimentally and theoretically. One of the aims is to construct tailored laser pulses that drive desired logic operations for quantum computation [1]. Two-dimensional (2D) nanodevices such as semiconductor quantum dots and rings are promising candidates for these applications due to their high flexibility in size, shape, and number of confined electrons [2].

Here we demonstrate that using quantum optimal control theory (OCT) [3] one can construct coherent single-electron quantum switches out of quantum rings (QRs) and double quantum dots (DQDs). Both of these systems are (in most cases) controllable so that full population transfer from one state into another can be achieved $[4,5]$. QRs and DQDs are routinely fabri-

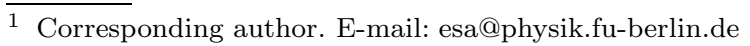

cated by, e.g., lithographic and etching techniques, and the decoherence times have been measured to be up to time scales of $10^{-6} \mathrm{~s}[6]$ and $10^{-7} \mathrm{~s}[7]$, respectively. Here we show that two-state switching processes such as flipping of the electron current in a QR and electron transport in a DQD can be achieved with a high precision (occupation $>0.99$ ) in only a few picoseconds. This is a significant improvement to the use of continuous waves (CWs) $[8,9]$.

OCT [3] is a powerful tool to find optimal laser pulses $\epsilon(t)$ driving the state $|\Psi(t)\rangle$ from a given initial state $\left|\Phi_{i}\right\rangle=|\Psi(t=0)\rangle$ to a target state $\left|\Phi_{f}\right\rangle$ in a finite time interval $T$. We point out that CWs with frequencies $\omega_{\text {if }}=\left(E_{\mathrm{f}}-E_{\mathrm{i}}\right)$ achieve accurate occupations only in special circumstances (e.g., $\pi$-pulses in two-level systems). Maximizing the overlap $\left|\left\langle\Psi(T) \mid \Phi_{\mathrm{F}}\right\rangle\right|^{2}$ and minimizing the fluence (time-integrated intensity) of the 
laser pulse leads, together with the time-dependent Schrödinger equation, to the control equations [4]

$$
\begin{aligned}
i \partial_{t} \Psi(t) & =\hat{H} \Psi(t), \Psi(0)=\Phi_{i}, \\
i \partial_{t} \chi(t) & =\hat{H} \chi(t), \chi(T)=\Phi_{f}\left\langle\Phi_{f} \mid \Psi(T)\right\rangle, \\
\epsilon(t) & =-\frac{A(t)}{\alpha} \operatorname{Im}\langle\chi(t)|\hat{\mu}| \Psi(t)\rangle,
\end{aligned}
$$

where $\chi(t)$ is the Lagrange multiplier, $\hat{\mu}$ is the dipole operator, $A(t)$ is the envelope function, and $\alpha$ is a constant penalty factor restricting the pulse intensity [10]. The control equations can be solved iteratively, and they converge monotonically towards the optimal laser pulse $\epsilon(t)[11,12]$. In the numerical calculations we have employed the octopus code [13].

The time-dependent Schrödinger equation of our 2D system confined in the $x y$ plane can be written as

$$
i \frac{\partial}{\partial t} \Psi(\mathbf{r}, t)=\left[\hat{H}_{0}-\hat{\boldsymbol{\mu}} \boldsymbol{\epsilon}(t)\right] \Psi(\mathbf{r}, t),
$$

where $\boldsymbol{\epsilon}(t)=\left(\epsilon_{x}(t), \epsilon_{y}(t)\right)$ is a two-component laser field propagating in $z$ direction. We apply the effective mass approximation for a GaAs semiconductor heterostructure: the effective mass is $m^{*}=0.067 m_{e}$ and the dielectric constant is $\kappa=12.7 \epsilon_{0}$. The results below are scaled to SI units accordingly. In the static Hamiltonian $\hat{H}_{0}=-\nabla^{2} / 2+V_{c}(\mathbf{r})$ we apply external potentials describing a $\mathrm{QR}$ and DQD respectively:

$$
\begin{gathered}
V_{c}^{\mathrm{QR}}(r)=\frac{1}{2} \omega_{1}^{2} r^{2}+V_{0} e^{-r^{2} / a^{2}} ; \\
V_{c}^{\mathrm{DQD}}(x, y)=\frac{1}{2} \omega_{2}^{2} \min \left[\begin{array}{c}
(x-d / 2)^{2}+y^{2}, \\
(x+d / 2)^{2}+y^{2}
\end{array}\right],
\end{gathered}
$$

with $r^{2}=x^{2}+y^{2}, \omega_{1}=10 \mathrm{meV}, V_{0}=200 \mathrm{meV}$, and $a=10 \mathrm{~nm}$, leading to a $\mathrm{QR}$ radius of $r_{0}=22 \mathrm{~nm}$. For the DQD we set $\omega_{2}=5.652 \mathrm{meV}$, and fix the interdot distance to $d=50.2 \mathrm{~nm}$. The external potentials are visualized in Fig. 1 together with the single-electron energy-level spectra. The QR states can be sorted according to the angular momenta $l$, for which we have the dipole selection rules $\Delta l= \pm 1$. The DQD states can be labeled as $|i j\rangle$, where $i=0,1, \ldots$ denotes the $(i+1)$ th bundle of states at $n \omega_{2}(n=1,2, \ldots)$ in the harmonic-oscillator limit $(d=0)$, and $j=0,1, \ldots$ denotes the $(j+1)$ th state in each bundle. Only transitions $i j \rightarrow(i \pm 1) j$ are allowed [5], i.e., those between the thickly marked (red) levels in Fig. 1(b).

In Fig. 2 we show the OCT procedure for the transi- (a)
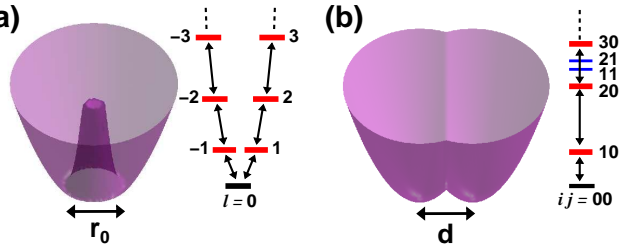

Fig. 1. Shape of the external potential describing a quantum ring (a) and double quantum dot (b), and a schematic plot of the lowest energy levels with the allowed transitions (arrows).

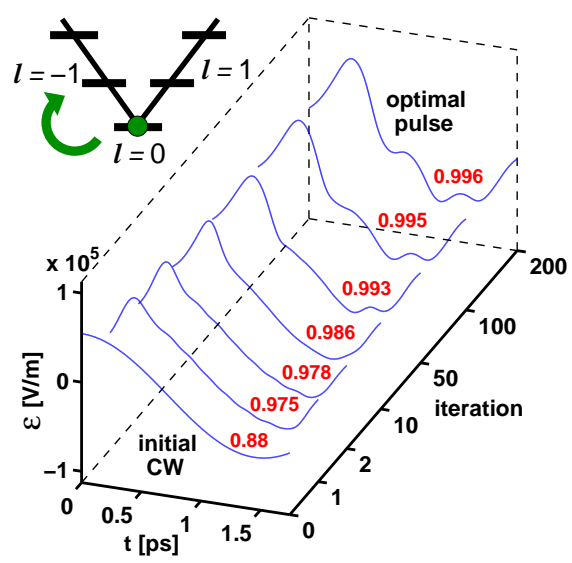

Fig. 2. Laser pulses ( $x$ components) during the iterative solving of the control equations for the transition $l=0 \rightarrow-1$ in a quantum ring. The initial pulse (zeroth iteration) is a continuous wave, and the optimal pulse is achieved after 200 iterations. The pulse envelope is $A(t)=e^{(t-T / 2)^{2} /(T / 4)^{2}}$ and the penalty factor is $\alpha=1$. The red numbers mark the target-state occupations achieved by the pulses.

tion $l=0 \rightarrow-1$ in a $\mathrm{QR}$. The pulse length is fixed to $T=1.75 \mathrm{ps}$. The initial pulse is a circularly polarized CW having a resonant frequency $\omega_{l=0}^{l= \pm 1}$ and amplitude $\Omega_{R} / \mu=\pi / \mu T$, where $\Omega_{R}$ is the Rabi frequency $(\pi$ pulse condition). The CW yields an occupation of 0.88 of the target state. The optimized pulses obtained by solving the control equations (1-3) with $\alpha_{0}=0.2$ and a Gaussian envelope function lead to occupations close to one after only a few iterations (see the red numbers in Fig. 2). At the end of the procedure we reach a maximum value of 0.996 . To obtain the same precision with a $\mathrm{CW}$ one would need to decrease the amplitude considerably in order to avoid the population flow into higher levels, and correspondingly increase the pulse length by a factor of around ten (see Ref. [4] for detailed 
(a) $l=1$
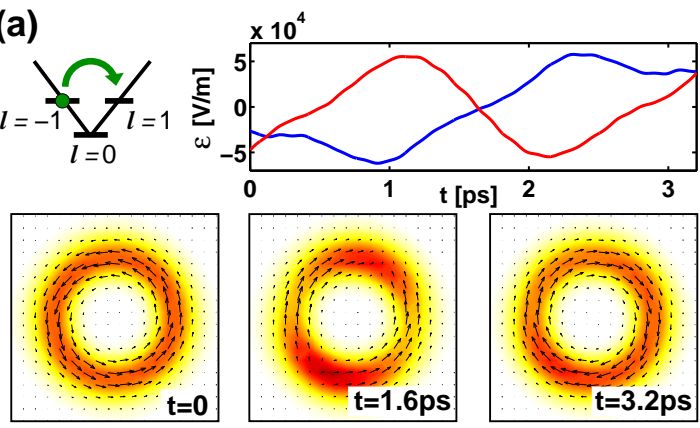

(b)

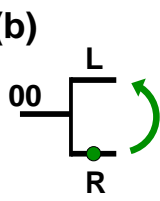

$\times 10^{4}$
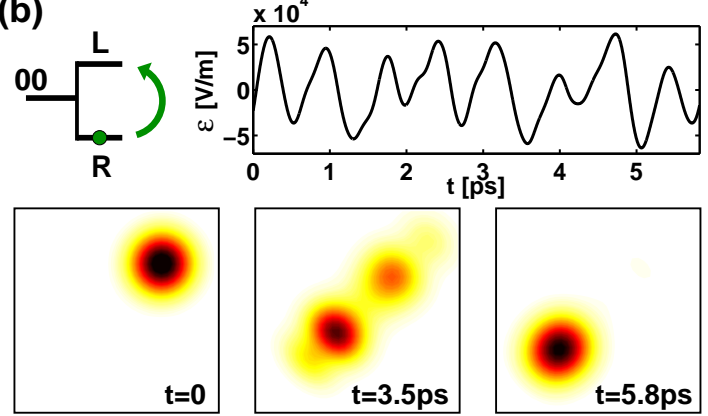

$t=0$
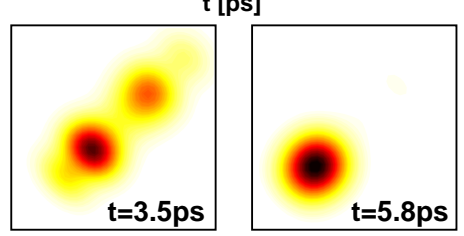

Fig. 3. Quantum ring as a magnetic switch (a) and a double quantum dot as a charge switch (b). The figure shows the optimized laser pulses $(A(t)=\alpha=1)$ and snapshots of the electron densities at different times in the transition. In the ring (a) the direction of the electron current changes, and in the double dot (b) the electron moves from right ( $\mathrm{R}$ ) into the left (L) well.

comparison). In the OCT approach, the higher-lying states are incorporated in the control problem, allowing the use of very short pulses with high precision.

In Fig. 3 we show two possible quantum switches made of a QR (a) and a DQD (b), respectively. In the QR we first initialize the system by exciting the electron from $l=0$ to $l=-1$. Then by optimizing the transition from $l=-1$ to $l=1$, the electron current (arrows), and thus the induced magnetic field, are flipped. Due to the high accuracy, the procedure can be repeated such that $l \leftrightharpoons-l$ operations are performed at desired times [4]. The corresponding magnetic switch of the induced field can be used to change the spin state of a subsystem placed in the middle of the ring, e.g., a magnetic particle [8] or an attached quantum dot. In the DQD instead, we first localize the electron in the right well $(\mathrm{R})$ by adding a small constant shift $(\Delta V \sim$ $2 \mathrm{meV}$ ) in the potential. Then we are able to transfer the electron to the left well $(\mathrm{L})$ in a few picoseconds.
By applying a reverse pulse we turn the it back again. Hence, we have a charge switch which, with respect to $10^{-6} \ldots 10^{-7}$ s decoherence times $[6,7]$, stays coherent for up to $\sim 10^{5}$ operations. The OCT procedure is insensitive to anharmonicities in the external potential and to the interdot distance $d[5]$.

In practical applications the shape of the confining potential $V_{c}$ should be estimated as precisely as possible, so that the optimized pulses could achieve a large number of successive switching operations with high fidelity. It has been shown that $V_{c}$ can be inverted from the single-electron transport spectrum with a reasonable accuracy [14]. Regarding the optimal laser pulses, we point out that the required picosecond pulse lengths, $\mathrm{THz}$ frequencies, and intensities of $\sim$ $10^{12} \mathrm{~W} / \mathrm{cm}^{2}$ suggested in this work are experimentally accessible using various laser technologies [15]. Precise pulse shaping of such pulses is the remaining challenge, but transient polarization grating, for example, shows already promising achievements in that direction [16].

This work was supported by EC FP6 through the Nanoquanta NoE (NMP4-CT-2004-500198), SANES project (NMP4-CT-2006-017310), DNANANODEVICES (NIST-2006-029192), Barcelona Supercomputing Center, the Humboldt Foundation, the Academy of Finland, and the Deutsche Forschungsgemeinschaft through SFB 658.

\section{References}

[1] See, e.g., D. P. Di Vincenzo and C. H. Bennett, Nature (London) 404, 247 (2000).

[2] For a review, see, e.g., L. P. Kouwenhoven, D. G. Austing, and S. Tarucha, Rep. Prog. Phys. 64701 (2001); S. M. Reimann and M. Manninen, Rev. Mod. Phys. 741283 (2002).

[3] A. P. Peirce, M. A. Dahleh, and H. Rabitz, Phys. Rev. A 37, 4950 (1988); R. Kosloff, S. A. Rice, P. Gaspard, S. Tersigni, and D. J. Tannor, Chem. Phys. 139, 201 (1989).

[4] E. Räsänen, A. Castro, J. Werschnik, A. Rubio, and E. K. U. Gross, Phys. Rev. Lett. 98, 157404 (2007).

[5] E. Räsänen, A. Castro, J. Werschnik, A. Rubio, and E. K. U. Gross, submitted (arxiv.org/abs/0707.0179).

[6] H. A. Engel, L. P. Kouwenhoven, D. Loss, and C. M. Marcus, Quantum Inf. Process. 3, 115 (2004).

[7] J. Gorman, D. G. Hasko, and D. A. Williams, Phys. Rev. Lett. 95, 090502 (2005). 
[8] Y. V. Pershin and C. Piermarocchi, Phys. Rev. B 72, 245331 (2005).

[9] M. Førre, J. P. Hansen, V. Popsueva, and A. Dubois, Phys. Rev. B 74, 165304 (2006).

[10] K. Sundermann and R. de Vivie-Riedle, J. Chem. Phys. 110, 1896 (1999).

[11] W. Zhu and H. Rabitz, J. Chem. Phys. 109, 385 (1998).

[12] J. Werschnik, Quantum Optimal Control Theory: Filter Techniques, Time-Dependent Targets, and TimeDependent Density-Functional Theory, (Cuvillier, Göttingen, 2006).

[13] A. Castro et al, Phys. Stat. Sol. (b) 243, 2465 (2006).

[14] E. Räsänen, J. Könemann, R. J. Haug, M. J. Puska, and R. M. Nieminen, Phys. Rev. B 70, 115308 (2004).

[15] J. H. Posthumus, Rep. Prog. Phys. 67, 623 (2004).

[16] A. G. Stepanov, J. Hebling, and J. Kuhl, Opt. Express 12, 4650 (2004). 